\title{
La enseñanza de la Geografía mediada por tecnologías en tercer nivel de Educación Primaria en el marco del Plan Ceibal (*)
}

Rosana Martínez Barcellos**

\section{Resumen}

Este trabajo se basa en una investigación realizada entre 2009 y 2010 en la que se abordan temas que hacen a la agenda didáctica de los maestros de Escuelas Públicas uruguayos.

Aportes recientes sobre el uso de las TIC en la enseñanza, así como los de la didáctica de la Geografía son el sustento teórico que enmarca nuestra investigación.

La enseñanza de la Geografía, su abordaje a partir del ingreso del Plan Ceibal a las aulas y el análisis de dichas prácticas, fueron parte de la construcción de una mirada sobre las estrategias de enseñanza que diseñan los docentes en Escuelas Primarias de nuestro país.

Algunas conclusiones obtenidas dan cuenta de una situación variopinta en las aulas. Las decisiones metodológicas que toman los docentes, referente a la dupla, TIC - Geografía, están estrechamente relacionadas a su formación y al deseo. Oportunidades y obstáculos se generan en dichos escenarios.

PALABRAS CLAVE: Educación, tecnologías, estrategias de enseñanza, Geografía, CEIBAL.

\section{Abstract}

This work is based on a research carried out between 2009 and 2010 and it deals with topics related to the mainstream classroom teachers at elementary school level.

The teaching of Geography, its methodology since Plan Ceibal was launched into the classes and the analysis of its teaching practices were part of the construction of a view over the teaching strategies designed by the primary school teachers in our country.

Some conclusions obtained informed about a wide range of situations in our classrooms. Teachers make methodological decisions about the couple ITC-Geography based on their education and desire. Different opportunities and obstacles are generated within these scenes.

KEYWORDS: Educations, technology, teaching strategies, Geography, CEIBAL.

\section{Consideraciones teóricas}

El artículo se basa en una investigación enfocada en el Proyecto de Conectividad Educativa de informática Básica para el Aprendizaje en Línea (Ceibal) y sus implicancias en el campo de la enseñanza de la Geografía. Este Plan está en marcha en el seno de la Educación Primaria, y a comienzos del 2011 inició una nueva fase en Educación Media. Los principios básicos de este Proyecto son la "igualdad en la oportunidad en el acceso a la tecnología, democratización del conocimiento y potenciación del aprendizaje" (Ceibal, Proyecto Pedagógico. 2007). Incluye la entrega de una computadora portátil, $\mathrm{XO}$, para cada niño y maestro de la Educación Primaria, en la esfera pública, ya que a nivel privado no ha llegado a extenderse de forma masiva.

El Proyecto tiene sus raíces en la Fundación OLPC cuyo precursor es Nicholas Negroponte, quien en el año 2005, presentó ante el Foro Económico Mundial de Davos, la voluntad de fabricar computadoras portátiles a costos muy bajos para implementar un proyecto educativo mundial.

Entre sus fundamentos, el Proyecto Ceibal esgrime aspectos expuestos en la Cumbre 
Mundial de la Sociedad de la Información de Ginebra del año 2003; en ellos expresa que las tecnologías de la información y las comunicaciones (TIC) son de valor crucial en el desarrollo y bienestar de la humanidad y que debido a su constante progreso, han brindado la posibilidad de acortar las brechas existentes en el acceso a la información en dos aspectos importantísimos: el tiempo y la distancia, las cuales cobran una dimensión diferente a la tradicional.

Partimos de la premisa de que enseñar es crear, hacer, convencer; es una actividad que implica acción y reflexión y pone en juego nuestras experiencias, concepciones, virtudes -y las que no lo son-, pero sobre todo, es un camino que hay que recorrer en compañía.

Hoy ese camino ha dado giros que nos obligan a volver sobre nuestras prácticas para evaluar si el mapa que nos hemos trazado nos lleva a destino correcto. Los viejos modelos de enseñanza ya no satisfacen a los niños y niñas. "Hay que reinventar la enseñanza escolar", dicen Levis y Gutiérrez Ferrer (2000), con el fin de que la institución "escuela" le dé las garantías a todos los individuos de acceder a una formación básica, sin importar nivel social o económico. Sólo se logrará esto si la escuela se adapta a los cambios vertiginosos que se dan en el mundo, como el avance que han tenido las TIC en la vida de las aulas, y despeja su vista para ver que "muchas de las teorías del pasado han perdido sentido" (Levis. Gutiérrez. 2000, 105).

Nos propusimos observar y analizar las prácticas de enseñanza de la Geografía mediadas por tecnologías. Para ello consideramos importantísimo reflexionar sobre los aspectos morales que rodean a la tarea de enseñar.

Eggen y Kauchak (2000) expresan al respecto: "Los objetivos que elegimos, las estrategias que usamos para alcanzar esos objetivos, la manera de relacionarnos con los alumnos, todo depende de lo que traemos al aula como seres humanos". (Eggen y Kauchak, 2000,17).

Diseñar estrategias de enseñanza en un mundo que no es igual al de ayer, y donde la velocidad de los cambios y la globalización de la información supera nuestra imaginación, hacen de la enseñanza de la geografía un desafío. Las TIC, "acercan a los estudiantes otras realidades, nuevas problemáticas sociales, territoriales, ambientales que no se pueden interpretar ni explicar a partir de los clásicos contenidos de la asignatura"(Zenobi, 2009, 108).

En la época actual, hemos asistido a fenómenos que cambiaron de una manera muy significativa los modos de pensar y comprender el mundo. La sociedad posindustrial y la cultura posmoderna reinantes, han traído cambios en los modos de percibir el mundo; cambios sesgados por el fenómeno de la globalización y las cada vez más fuertes tecnologías de la información y la comunicación, siendo estos factores fundamentales en la "evolución social de la población. En este marco, el desarrollo tecnológico ha obtenido una alta valorización y se ha convertido en ideal para que la ciencia se presente como aplicación concreta a fin de satisfacer las demandas sociales de dinámica cambiante. Por lo tanto, el medio tecnológico y particularmente en su interior las tecnologías de la información, pueden considerarse actualmente el sustento de las actividades humanas sobre el planeta" (Buzai, 2004, 21).

Buzai (2004) afirma que casi todas las actividades sociales y culturales de las personas están vinculadas de algún modo con las TIC. En este panorama el autor desarrolla las relaciones entre la Geografía y la Informática para el análisis del espacio, en un "nuevo ambiente" que permitirá crear una nueva mirada sobre la realidad.

La educación no es ajena a ella y ha tenido que sufrir modificaciones y adaptaciones que devienen de esta realidad. Las formas de abordaje de la Geografía han variado de acuerdo a las concepciones de la enseñanza, del aprendizaje y de la geografía reinantes, pero también son afectadas por otros fenómenos, como el desarrollo inconmensurable de las TIC. Hasta 
hace algunos años no vislumbrábamos la posibilidad de acceder a mapas interactivos, o de que existirían fotografías aéreas y satelitales de casi todos los lugares imaginados, o las visualizaciones tridimensionales entre otras. Hoy las TIC nos brindan posibilidades de conocer el espacio de maneras diferentes. Las estrategias para enseñar los conceptos geográficos son diferentes. Descubrámoslas.

\section{Metodología}

Los sustentos metodológicos de este trabajo, son los que rigen a la investigación cualitativa en un marco fenomenológico. Entendiendo por investigación cualitativa aquella investigación que en función de acercamientos al objeto de estudio, genera datos de tipo descriptivos (Taylor y Bogdan. 1987) apoyada en una perspectiva fenomenológica que significa la interpretación de los fenómenos sociales y como tal, la educación. Estos fenómenos se captan a través de lo que los individuos hacen, dicen, actúan; interpretar esto es nuestra labor.

El diseño metodológico implicó una serie de decisiones que dieron como resultado un mapa con el cual nos guiamos a lo largo de este recorrido. "Diseñar significa ante todo, tomar decisiones a lo largo de todo proceso de investigación y sobre todas las fases o pasos que conlleva dicho proceso. Algunas de estas decisiones se tomarán al principio, mientras se va perfilando el problema a investigar y se delimitan los casos, el tiempo y el contexto de estudio. Otras irán surgiendo sobre la marcha"Valles $(2003,78)$. Este diseño, de corte cualitativo, tiene la cualidad de ser flexible por lo que nos dio la posibilidad de modificar nuestras decisiones de acuerdo a lo que fue surgiendo en el campo.

El objetivo planteado fue observar prácticas de enseñanza de la Geografía en las cuales se emplearan las TIC a través del uso de las XO, para reflexionar sobre ellas a la luz de las teorías y poder construir un marco referencial que diera cuenta de las estrategias de enseñanza y sus sustentos, en el camino de las buenas prácticas de un campo que ha comenzado a emerger en la educación primaria con la implementación del Plan Ceibal.

Se seleccionaron docentes de quinto y sexto año de Educación Primaria ya que existen aspectos importantes en cuanto a la construcción de conceptos geográficos de acuerdo a las posibilidades empíricas de estos grupos. Se trabajó con cinco docentes de escuelas de Montevideo, cuatro docentes de San José, tres de Florida, una docente de Canelones y dos de Colonia.

Como decíamos al comienzo de este trabajo, el Plan Ceibal fue implementado en varias etapas y consideramos de suma importancia para la elaboración este trabajo, contar con datos provenientes de los primeros actores que inauguraron este capítulo en la Educación Pública uruguaya. Para acceder a una Escuela de Florida, participante del piloto realizado por el Plan Ceibal, elevamos varias notas solicitando autorización. Luego de unos meses, recibimos la llamada del director de la escuela, autorizando nuestra entrada a la institución.

Otros informantes calificados fueron seleccionados por su grado de implicancia con el campo de conocimiento Geografía: Una docente (M10) de Colonia quien participó junto a la maestra de Montevideo, M1, en la elaboración del libro de texto de Ciencias Sociales y además es maestra de Apoyo al Plan Ceibal; y una profesora de Geografía del mismo departamento (quien trabaja a nivel escolar en el ámbito privado, asesorando a docentes sobre su área, y en Secundaria, en cuyas aulas comenzó a utilizar las laptops del Plan Ceibal).

En un primer momento del diseño metodológico, nos habíamos propuesto realizar entrevistas, 
observaciones y análisis de documentos pero la falta de disposición en algunos docentes para permitirnos el acceso a estos documentos nos llevó a utilizar como técnicas de relevamiento de datos, la observación y la entrevista simplemente.

Desde el comienzo del trabajo de campo se fueron tratando los datos con determinados cuidados, registrando y analizando continuamente lo que surgía de las técnicas aplicadas. Se realizaron transcripciones de las grabaciones y a partir de codificación de información, análisis e interpretación de los datos fuimos construyendo tablas que nos sirvieron para organizar los hallazgos y a la luz de nuestro marco teórico fueron surgiendo una serie de categorías y conclusiones que constituyen los resultados de esta investigación.

\section{Técnicas}

Las técnicas seleccionadas para la obtención de datos y el análisis de las prácticas fueron:

- La observación de clases de Geografía. En cuanto a esta técnica Pardinas (1996) dice: "Observación es la acción de observar, de mirar detenidamente. Pero este primer significado de la palabra se presta, en el trabajo científico, a una ambigüedad que es necesario disipar desde un principio. La observación puede ser estudiada desde el investigador, que mira detenidamente y desde lo observado, lo mirado detenidamente... pero observación significa también el conjunto de cosas observadas, el conjunto de datos y el conjunto de fenómenos..." (Pardinas, 1996, 89). Según este autor generalmente, nos consideramos buenos observadores pero esto no es tan sencillo como parece. Debemos abandonar nuestra subjetividad para ser observadores precisos del fenómeno a estudiar. Para esto empleamos la observación simple, no participante.

Durante este proceso pusimos especial atención a las estrategias que utiliza el docente para enseñar Geografía mediada por las tecnologías de la información y comunicación, se analizarán los elementos que integran estas estrategias y cómo el docente utiliza estos medios para favorecer los aprendizajes de los conceptos geográficos en los niños.

La observación, de acuerdo con Gimeno Sacristán y Pérez Gómez (1995), "supone estancias prolongadas del o los investigadores en el medio natural, observando ... directamente o no, en la vida del aula, para registrar los acontecimientos, las redes de conducta, los esquemas de actuación comunes o singulares, habituales o insólitos..."(Gimeno y Pérez, A. 1995, 126-127)

- Una segunda técnica para la obtención de datos sobre el problema identificado, es la entrevista. Al respecto, Valles $(2003,179)$ cita a Erlandson y otros: “... Las entrevistas pueden adoptar una variedad de formas, incluyendo una gama desde las que son muy enfocadas o predeterminadas a las que son muy abiertas (...) La más común es la entrevista semiestructurada que es guiada por un conjunto de preguntas y cuestiones básicas a explorar, pero ni la redacción exacta, ni el orden de las preguntas está predeterminado (...)" (Valles, 2003 , 179). A través de las entrevistas intentaremos comprender los modos de actuar, sentir y pensar de los participantes, al igual que nos permitirá indagar sobre los elementos subyacentes a las prácticas, que no se dicen a la hora de enseñar pero dan sentido a las mismas. Del mismo modo trataremos de comprender los procesos que se dan en la otra parte del triángulo didáctico. "La enseñanza está concebida como una actividad que implica el tratado conjunto de profesores y estudiantes. El trabajo implica el ejercicio tanto del pensamiento como de la acción por parte de todos los participantes. Además, los enseñantes aprenden y los alumnos enseñan. Ambas funciones de cada acto pueden considerarse como parte fundamental de la investigación" (Wittrock, 1989, 20).

A algunos docentes, se realizaron entrevistas en más de una oportunidad. Generalmente se 
fijó un día, previo a la observación, para realizar una entrevista en profundidad.

Se realizaron un total de quince entrevistas y diez observaciones.

A partir de estas técnicas se apeló a la triangulación que, a decir de Gimeno y Pérez (1995), es "el contraste plural de fuentes, métodos, informaciones, recursos. Su objetivo es provocar el intercambio de pareceres o la contrastación de registros o informes. Comparar las diferentes perspectivas de los diversos agentes con las que se interpretan los acontecimientos del aula es un procedimiento indispensable, tanto para clarificar las distorsiones y sesgos subjetivos que necesariamente se producen en la representación individual o grupal de la vida cotidiana del aula, como para comprender el origen y proceso de formación de tales representaciones subjetivas, ofreciendo la posibilidad ... de interpretaciones distintas ... enriquecer y ampliar el ámbito de la representación subjetiva y construir más críticamente su pensamiento y su acción" (Gimeno y Pérez, 1995, 127).

\section{Resultados de la investigación}

\section{4.a. Primeros hallazgos}

En este trabajo de investigación hemos indagado sobre las prácticas de enseñanza de la Geografía mediadas por tecnología. Lejos estamos de responder todas las preguntas que surjan en torno al tema pero intentamos describir escenarios y actores, en los que, y por los que, se llevaron a cabo determinadas prácticas de enseñanza.

En estos escenarios, escuelas públicas de nuestro país, observamos los pasos estratégicamente pensados, que dieron los docentes para aproximarse a sus objetivos de enseñanza de la Geografía. Estos actores, protagonistas de nuestra investigación, nos dejaron ser parte de su mundo, permitiéndonos ver aquellos aspectos que afectan y determinan sus prácticas de enseñanza.

Para interpretar los datos que surgieron del trabajo de campo, enfocamos el análisis en la integración de las TIC para la enseñanza de la Geografía en las clases de quinto y sexto año de Educación Primaria, ajustándonos a nuestro marco teórico de referencia y dándonos el permiso de aportar nuestra mirada como investigadores.

Los primeros acercamientos al campo dejaron impresiones en el mapa que fuimos construyendo a medida que avanzábamos.

En primer lugar queremos describir algunos elementos que surgieron de las entrevistas realizadas. De acuerdo a lo que expresaban los entrevistados, pudimos ir haciendo algunas clasificaciones y agrupaciones que nos permitieron organizar la información que aportaban. Esto fue posible a través de una lectura y relectura de las transcripciones de las entrevistas, seleccionando ideas que en ellas surgían de diversas formas y agrupándolas para ir estableciendo algunas relaciones en torno al tema que investigamos.

Las entrevistas fueron realizadas basándose en un guión que apuntaba a obtener información referida a distintos aspectos: el docente y las tecnologías en el aula; el uso educativo de las XO; las estrategias de enseñanza de la Geografía y la mediación tecnológica. En el primer eje, pudimos discriminar algunos elementos que aludían a la formación docente, los que agrupamos de la siguiente manera: actualización en la didáctica de las disciplinas, y formación en el uso educativo de las TIC.

De acuerdo a la información obtenida, los docentes entrevistados en su mayoría han realizado cursos de actualización en la didáctica de las disciplinas, o están realizando alguno. Dentro de los cursos que mencionaron encontramos el Curso de Maestros Adscriptores, el cual consta de cinco módulos que abarcan la didáctica de las distintas disciplinas (Matemáticas, Lenguaje, 
Educación Artística, Ciencias Sociales, Ciencias Naturales). Éste lo realizaron las cinco docentes de Montevideo, las dos docentes de San José de Mayo y la maestra de Colonia. Cabe resaltar que estas docentes viven y se desempeñan en las capitales departamentales. Sin embargo, las docentes de Florida, y de Canelones no realizaron este curso, pero lo adjudicamos al tipo de escuela ya que no eran de práctica ninguna de las dos. Curso de Áreas Integradas específico en Ciencias Sociales expresaron haberlo hecho las maestras: M1, M2, M3, M6, M10. Luego mencionan otros cursos que refieren generalmente a didáctica. Se observa interés en realizar todo tipo de capacitaciones.

Si bien, desde el punto de vista didáctico, casi todos los docentes han recibido algún tipo de actualización y buscan estar en continua formación, observamos en estas entrevistas que no han mencionado cursos específicos de didáctica de la Geografía, sí se mencionan de Ciencias Sociales en los que se integran todos los campos de conocimiento pertenecientes a esta área (Historia, Geografía, Ética y Derecho).

Por otro lado, frente a la implementación del Plan Ceibal, surge reiteradamente, la ausencia de cursos referidos al uso educativo de las TIC. En la siguiente tabla podemos ver la cantidad de docentes que realizaron cursos previos a la llegada de las XO para sus alumnos, los que realizaron cursos luego de ello, y los que aún no tienen cursos. En todos los casos, nos encontramos con docentes que desarrollaron estrategias de aprendizaje de la herramienta a través del intercambio con sus compañeros:

\section{Cursos vinculados a las XO}

Tabla 1 Cursos de formación en el uso de las XO.

\begin{tabular}{|c|c|c|c|c|c|c|}
\hline Maestra & DEPARTAMENTO & $\begin{array}{c}\text { ETAPA EN } \\
\text { QUE SE } \\
\text { IMPLEMENTÓ } \\
\text { EL PLAN }\end{array}$ & $\begin{array}{c}\text { Realizaron } \\
\text { cursos } \\
\text { OFICIALES } \\
\text { de formación } \\
\text { vinculados al } \\
\text { uso de las XO } \\
\text { previos a la } \\
\text { llegada de las } \\
\text { mismas }\end{array}$ & $\begin{array}{l}\text { Realizaron } \\
\text { cursos } \\
\text { OFICIALES } \\
\text { vinculados al } \\
\text { uso de las XO } \\
\text { luego de la } \\
\text { llegada de las } \\
\text { mismas. }\end{array}$ & $\begin{array}{l}\text { Sin } \\
\text { cursos } \\
\text { aún. }\end{array}$ & $\begin{array}{l}\text { Crearon } \\
\text { estrategias de } \\
\text { autoformación } \\
\text { y aprendizaje } \\
\text { colaborativo } \\
\text { entre docentes }\end{array}$ \\
\hline M1 & \multirow{5}{*}{ MONTEVIDEO } & \multirow{5}{*}{3} & NO & $\mathrm{SI}$ & - & $\mathrm{SI}$ \\
\hline M2 & & & $\mathrm{NO}$ & $\mathrm{SI}$ & - & SI \\
\hline M3 & & & $\mathrm{NO}$ & $\mathrm{SI}$ & - & $\mathrm{SI}$ \\
\hline M4 & & & NO & $\mathrm{NO}$ & $\mathrm{SI}$ & $\mathrm{SI}$ \\
\hline M5 & & & $\mathrm{NO}$ & $\mathrm{NO}$ & $\mathrm{SI}$ & $\mathrm{SI}$ \\
\hline M6 & SAN JOSÉ & 2 & NO & NO & $\mathrm{SI}$ & $\mathrm{SI}$ \\
\hline M7 & FLORIDA & PILOTO & $\mathrm{NO}$ & $\mathrm{SI}$ & - & SI \\
\hline M8 & & & $\mathrm{NO}$ & SI & - & $\mathrm{SI}$ \\
\hline M9 & CANELONES & 2 & NO & $\mathrm{NO}$ & $\mathrm{SI}$ & SI \\
\hline M10 & COLONIA & 2 & $\mathrm{NO}$ & SI & - & $\mathrm{SI}$ \\
\hline MAPC & SAN JOSÉ & 2 & NO & NO & $\mathrm{SI}$ & $\mathrm{SI}$ \\
\hline MPG & SAN JOSÉ & 2 & NO & NO & SI & SI \\
\hline
\end{tabular}


Las maestras que participaron de las primeras etapas de funcionamiento del Plan Ceibal expresaban:

"En realidad, a nosotros nos dieron las máquinas el jueves a los maestros, y el lunes se la daban a los niños, tuvimos un fin de semana para ver qué hacer. Vinieron un par de veces las maestras de tecnología de inspección, que estaban acostumbradas a trabajar con máquinas grandes, pero fue poco lo que aprendimos porque ellas tampoco sabían el manejo..." (M8)

"Acá nos reunimos y nos ayudábamos." (M7)

Los elementos que surgieron reiteradamente en las entrevistas nos permiten afirmar que existe la imperiosa necesidad de actualización y formación docente en los temas referidos a la integración de las tecnologías a la enseñanza y apoyo en cuanto a aspectos disciplinares y didácticos de la Geografía. Las experiencias que pudimos recopilar en este trabajo dejan en claro que si bien hay docentes que buscan estar en constante actualización como forma de mejorar sus prácticas, también hay quienes a pesar de reconocer la necesidad y desear acceder a este tipo de formación, no pueden hacerlo, ya sea por la falta de ofertas de cursos o por la centralización de este tipo de actividades que generalmente se da en las capitales departamentales.

No fue nuestro objetivo analizar estas situaciones pero son dignas de atención, quizá en futuras investigaciones, ya que como expresan Gimeno y Pérez (1995) "Lo que se enseña, se sugiere o se obliga a aprender, expresa los valores y funciones que la escuela difunde en un contexto social e histórico concreto" (Gimeno y Pérez 1995, 172). Es en este contexto en donde el maestro debe cumplir con determinadas exigencias pautadas por las necesidades sociales, institucionales y personales. Se espera de él que todo lo sepa, que todo lo pueda resolver.

Por otro lado, hemos podido extraer de las entrevistas una autoformación que caracteriza al "estilo de aprendizaje" de algunos maestros, tanto de lo disciplinar como de lo tecnológico que necesitan para elaborar estrategias.

"Yo creo que desde lo disciplinar está siendo siempre autodidacta el docente..." (MPG)

"Lo que yo sí pienso es que previo a la entrega de las $\mathrm{XO}$, tendríamos que haber tenido nosotros una formación porque ahí ha sido investigar, ensayo y error." (MPG)

"Primero que nada, me gusta. Conozco la escuela, o a la mayoría de los niños, entonces era un desafío para todos. Y, tengo un gran apoyo de la maestra de quinto que sabe mucho. Por gusto también ha aprendido mucho y nos intercambiamos. Ha sido a golpe y porrazo. Lamentablemente ha sido asi". (MAPC)

Es así que encontramos maestros que por sus posibilidades de acceso a la formación, se transforman en "autodidactas", como expresaba la maestra de San José (MPG) y que transcribíamos en párrafos anteriores. La maestra se refiere a la autoformación en el plano disciplinar; ella nos decía que desde lo didáctico siempre existe apoyo desde los directores e inspectores o a través de cursos, pero en lo específico de cada disciplina, el maestro debe buscar la forma de acceder al conocimiento prácticamente solo. Si bien este estilo "autodidacta" se da en lo disciplinar, podemos decir que también se da en lo referido al manejo de las XO; en varias de las entrevistas realizadas encontramos que los docentes tuvieron que aprender el manejo de la herramienta tecnológica para poder incorporarla a sus clases sin mediación formal de parte de las instituciones que están a cargo del Plan Ceibal (M4, M5, M6, M9).

“... el día que nos entregaron las $X O$, que éramos no sé si 160 personas o algo así, en un patio de una escuela, un patio cerrado. Nos entregaron la $X O$, nos hicieron ahí algunas indicaciones, después nos repartieron en grupos de 30 o 40 en un salón y estuvimos con una persona, "Amigo del Plan Ceibal", que, en dos horas, con 40 personas, con una computadora... Lo que hicimos fue poner el nombre, elegir el color, sacar algunas fotos. ¡Salimos chochas por lo que sabíamos hacer! ¡No sabíamos nada!" (M6). 
Otros indicios de esto: el "no rotundo" que expresaba la maestra M7 cuando contestaba sobre la capacitación que no tuvieron previamente a la llegada del Plan Ceibal a su escuela; el aprender a "ensayo y error" que planteaba la maestra, MPG; o los problemas que veía la maestra M4 cuando nos contaba "enseguida hubo el problema de que no estábamos formados nosotros para poder utilizar la herramienta como se debería porque no teníamos ningún curso a la vez que la implementación".

En otros casos nos encontramos con experiencias de docentes que plantearon resistencias, apatía o temor ante las TIC en el aula. Buckingham (2008) plantea que muchas veces, la apatía de los docentes ante las tecnologías se debe a que no tienen la capacitación que requieren para manejarlas y usarlas en la enseñanza. Reafirmando lo que decíamos, transcribimos lo que expresaban nuestros entrevistados:

"Bueno mirá, acá tengo muchas cosas para hacer y el Ceibal para mí no es una prioridad" (MDPC).

"Pero no hubo cursos ni nada de capacitación. Los cursos empezaron, después, al tiempo después que nosotros ya teníamos las máquinas... cuando la recibimos nosotros, no había nada. Cursos formales no. Entonces... fue difícil arrancar. La carreta adelante de los bueyes, primero la máquina y después ver como se usa". (M8)

"Vos sabés que en esta escuela se ha dado que hay algunos docentes que están todavía un poco reticentes como que le disparan un poco, ahora que surgió esto del apoyo están un poco contentos porque se sienten "qué suerte porque yo no sé nada de esto". Pero bueno... Como que al estar ahí encerrados no quieren que vos le expliques mucho al principio, muy cerrado. Después, como de a poquito se fueron abriendo. Pero ya te digo la mitad de los docentes estaban, sobre todo las maestras con más años en la escuela como que estaban más cerradas". (M8).

En algunos casos esto generó resistencias, desinterés y decepción, pero en otros casos los docentes generaron estrategias que les permitieron incluir el recurso en sus prácticas. Tal es el caso que nos relataba el Director de una de las instituciones visitadas, cuando nos contaba sobre una maestra que en un primer momento decidió jubilarse porque creía que no iba a poder manejar las computadoras, pero poco a poco fue haciendo suyo esta herramienta hasta tal punto, que es una de las maestras que actúan como potenciadoras en el uso de las XO en la institución.

Si bien hay docentes que se resisten, también hay quienes encuentran una oportunidad en cada instancia de actualización y formación profesional en el manejo de la herramienta tecnológica.

"Y hay gente... que aceptó el desafío, que aceptó la capacitación que además lo ve como realmente, algo bueno, algo que puede cambiar..." (MDPC).

A través de los párrafos anteriores pudimos ver cómo surgieron algunos elementos relacionados a la formación, pero a partir de la teoría y los datos podemos afirmar que las estrategias de enseñanza no se fraguan en la técnica o en la teoría, sino que tienen un gran componente ético y moral. Hay docentes que han declarado su "ignorancia tecnológica" pero que a pesar de sus dificultades han podido diseñar y llevar a cabo con éxito, estrategias de enseñanza de la Geografía mediadas por éstas. "El quid del cambio es cómo asumen los individuos esta realidad" (Fullan, 2002,61). 
Uno de los propósitos que nos habíamos planteado en este trabajo fue describir las estrategias de enseñanza de la Geografía mediadas por la tecnología, pero consideramos que hay una serie de variables que afectan directamente esas prácticas de enseñanza y que deben ser tenidas en cuenta para comprenderlas. La cantidad de computadores averiados, el tiempo de carga de la batería, el estado de la instalación eléctrica de las escuelas y aspectos edilicios, son algunas de estas variables.

Algunas problemáticas con las que nos encontramos fueron: el alto porcentaje de computadores averiados, en algunas clases; el tiempo de carga de la batería y la falta de tomas de corriente adecuadas para realizar la carga en clase, si era necesario. Muchas veces el maestro planificaba determinadas actividades que requerían mayor tiempo del que tenían carga las baterías, lo que impedía terminar la actividad y muchas veces generaba desmotivación en los alumnos. Concomitantemente, el tiempo que requería resolver determinadas situaciones propuestas por el docente utilizando las $\mathrm{XO}$, generalmente era mayor a lo que les llevaba realizarlas por otras vías. Frente a esto manifiestan que han desarrollado la capacidad de saber esperar, de elasticidad en las propuestas.

"Del año pasado a este he visto que tenemos más paciencia....Este año decís: Bueno, si ya no funciona lo dejamos y lo vemos en otro momento. Lo probamos de nuevo o lo pruebo yo mientras ellos están en otra cosa. De manera que los tiempos que nos apremian no se vean perjudicados" (M1).

Nos animamos a afirmar que esta variable, en algunos casos, es determinante en la consolidación de las estrategias de enseñanza con las tecnologías, ya que al no concretarse los objetivos en el tiempo planificado y pautado por la institución, puede provocar la "deserción tecnológica" de los docentes en el sentido de que abandonan las prácticas que emplean las TIC como herramienta pedagógica para optimizar el uso de los tiempos.

"Es un obstáculo que los desmotiva a ellos y a los docentes, también" (M1)

Establecemos una analogía entre los docentes y los exploradores o navegantes: éstos manejan las cartas geográficas para aventurarse en un territorio que cambia constantemente, con el fin de interpretarlo, intervenir sobre él y sobre todo "descubrir", al igual que los docentes con todo su bagaje. Este bagaje personal que traen al aula- expresan Eggan y Kauchak (2000) que todas nuestras decisiones dependen de lo que somos como personas -, hace que se desplieguen estrategias que -según De la Torre (2000)-, se organizan de modo tal que favorecen el logro de las metas esperadas.

De las entrevistas realizadas interpretamos los modos de actuar de los docentes ante la toma de decisiones relacionadas a la enseñanza de la Geografía con mediación tecnológica:

"Docentes con mapa antiguo en ciudad moderna".

En este grupo de docentes ubicamos a aquellos que se aferran a las formas de enseñar que originalmente los modelaron.

Como en la obra pictórica de Johannes Vermeer ${ }^{1}$ (1669), "El geógrafo", donde éste intenta comprender lo que ve afuera a partir de su mapa, midiendo y comparando cada detalle, hay docentes que intentan repetir formas de enseñar que fueron eficaces, para un tiempo y espacio determinado, en un tiempo y espacio que está en continuo cambio. Donde hay necesidades diferentes y entornos diferentes. 
Por un lado, las tecnologías que irrumpen en el plano educativo y por otro los docentes que se resisten a ellas. Resistencia que puede atender a un sinfín de razones válidas o no, dependiendo de los lugares desde donde nos ubiquemos. Razones que atienden a cuestiones referidas a la formación en el uso de ellas, como veíamos anteriormente, o razones que impliquen decisiones de cuestión moral y ético.

“...yo enseñé, y considero que enseñé bien sin ella en 30 años, 31” (M6).

No por pertenecer a este grupo significa que los docentes no lleven a cabo "buenas prácticas". Un ejemplo de esto fue la maestra que citamos anteriormente. Maestra del departamento de San José que aceptó nuestra presencia en una de sus clases de Geografía.

En la clase, la maestra trabajó con el contenido "Globalización". Entregó a cada niño, envoltorios y recipientes representativos de marcas comerciales, sobre el pizarrón dispuso un planisferio y comenzó así una clase que desde el análisis didáctico y disciplinar podríamos llamarla "magistral". En ella generó diversas situaciones que provocaron la reflexión y construcción del concepto en los niños. Hubo momentos en donde la intervención del docente fue clave para dirigir la mirada de los niños a los elementos que ayudaron a construir el concepto.

Utilizó recursos valiosos para la enseñanza de la Geografía como el mapa o las preguntas que llevaban a la reflexión. Pero, para sorpresa nuestra, en ningún momento utilizó la XO.

¿Podría haber utilizado las TIC para enseñar este contenido? ¿Qué motivos llevaron a tomar estas decisiones?

La forma que desarrolló la clase, la intervención de los alumnos y la intervención docente, el logro de los propósitos para esta clase, nos confirman que fue una clase magistral no importa cuál haya sido el recurso utilizado o no utilizado.

A partir del análisis de los datos podemos afirmar que en esta maestra, la resistencia a los cambios es el factor determinante para seleccionar este tipo de estrategias y no otras. Continúa aferrada a prácticas que en sus 31 años de trabajo fue consolidando y a través de ellas alcanzó sus metas de enseñanza. No dejan de ser estrategias muy válidas, pero en estos procedimientos se ha dejado de lado un aspecto importantísimo, la cultura que impera en la sociedad actual, la cultura digital. Por lo tanto, la decisión de "abrir las puertas" a ella (Maggio, 2005) queda supeditada al docente.

La maestra de Florida, desde su doble rol de maestra de aula y maestra de apoyo al Ceibal nos contaba que el abrir la puerta del aula a una nueva forma de enseñar y al intercambio de experiencias, resultó bastante difícil para algunas maestras.

En otros casos, las resistencias se transforman en inacción. En este nivel encontramos a la maestra M2, quien nos abrió la puerta de su aula para observar una clase de Geografía pero el temor ante lo desconocido, la falta de conocimientos sobre las posibilidades que ofrece el recurso tecnológico, hizo que la docente se "paralizara". Le solicitó a la maestra dinamizadora del Plan Ceibal, que llevara adelante la clase porque no se sentía competente ante tal desafío.

Surge la siguiente pregunta: ¿Tenemos el derecho los docentes de decidir sobre esto o es una obligación? Amparándonos en el marco teórico que nos sustenta, la respuestano admite dudas: es una obligación.

Pero ante esta inminente responsabilidad nos preguntamos: ¿Los dispositivos desplegados para la preparación en el uso de las TIC, de los docentes que han desarrollado excelentes carreras, atienden sus necesidades? Son muchos más que éstos los docentes que se aferran a prácticas exitosas que han realizado a lo largo de muchos años de enseñanza, o a tecnologías tradicionales y que se resisten a transformar sus prácticas en pro de las nuevas tecnologías. 
Por un lado la experiencia de tantos años y por otro la velocidad de los cambios. ¿Cómo hacer para que esto no impacte tan fuerte en los docentes?

\section{4.b. "Imposibilidad de usar el mapa. ¿Perdidos?"}

En este grupo de docentes ubicamos a aquellos que manifiestan y reconocen la importancia de las TIC en la agenda didáctica, entienden que es necesario integrarlas a sus prácticas de enseñanza pero necesitan capacitarse en el uso y la aplicación didáctica de éstas. Expresan que necesitan ser enseñados también.

La importancia de la formación docente surge nuevamente pero desde un sentido más amplio. Los docentes se enfrentan a la necesidad de un mediador entre ellos y las TIC para hacer posible la integración de las TIC en sus propuestas didácticas.

"Hay maestros que tienen capacidad y lo hacen grandioso, pero hay muchos que no. Yo no soy una persona virtuosa para la tecnología y si no me explicas ciertas cosas, yo no sé dónde buscarlas siquiera. Porque también sé que hay un montón de actividades preciosas y también me gustaría buscar..." (M9).

Reiteradas veces escuchamos en las entrevistas que no sabían cómo usar la XO, que cuando llegaron a las aulas tuvieron que aprender con los niños, o con ayuda de un compañero quien, por distintos medios, había logrado avanzar en el conocimiento de la herramienta.

"Ahora es un poco complejo el aspecto técnico que es lo que más le cuesta el maestro incorporar" (MDPC).

"...donde podamos intercambiar lo que sabemos con el maestro y a su vez aprender con los niños. Que muchas veces, son los niños los que nos enseñan a nosotros" (MAPC).

Reconocen sus limitaciones y permiten al otro que oficie de guía en la esta situación de aprendizaje que deben transitar al enfrentarse a las tecnologías y a la pregunta sobre cuál es la forma de integrarlas genuinamente en sus prácticas. Esto implica adoptar una postura de humildad, reconocer que no se posee el saber a modo de oráculo y que es necesario aprender con el otro.

Por lo tanto, los docentes han desarrollado estrategias de enseñanza que implican sucesivos ensayos y requieren apoyos para poder consolidarse como potenciales prácticas de enseñanza de la Geografía.

Expertos navegantes.

En nuestros fundamentos teóricos planteábamos las ideas de Litwin (2008) que enuncian la concurrencia de buenas prácticas cuando, entre otros elementos importantes también, hay dominio disciplinar.

Para que estas construcciones sean válidas desde lo epistemológico y desde lo didáctico, consideramos que el dominio disciplinar debe darse en dos terrenos: en la disciplina en sí misma, la Geografía en nuestro caso, y en lo tecnológico. No es posible trazar la ruta de navegación didáctica si no se conocen las herramientas con las cuales leer los mapas que constituyen los contextos de enseñanza.

Bajo este subtítulo hemos ubicado a los docentes que además del dominio conceptual de la disciplina, han desarrollado diversas capacidades que les permiten ser hábiles usuarios de la tecnología, en el desarrollo de acciones que tengan como meta la concreción de los objetivos.

Aquí encontramos a docentes como las maestras: M1, M5, M10, M8, que si bien expresaron en la entrevista no haber tenido ningún tipo de capacitación formal previa en el manejo de las $\mathrm{XO}$, buscaron distintos caminos para que esa limitación no fuera impedimento para integrarlas a sus construcciones metodológicas. 
"Yo creo que primero fue romper estructuras, fue un quiebre total, porque teníamos eso formal y teníamos que adaptarnos. Que los chiquilines anduvieran caminando adentro del salón para un lado y para el otro. Cambiar la forma, ya la hemos cambiado otras veces, primero la pusimos en U para poder caminar y poder verlos a todos. El estar cambiando permanentemente el salón, que el movimiento ese no era falta de orden, de disciplina: era un desorden bueno. Pero eso a maestros con algunos años, cuesta más, muchísimo. Entonces, primero fue romper esas estructuras que fue horrible pero después, yo dije, qué privilegio que haya sido esta escuela. Porque a mí me obligó a reciclarme, a aggiornarme en un montón de cosas. Yo siempre que me obligan a ajironarme, primero rezongo, pero después agradezco." (M8)

“... también estábamos explorando las distintas posibilidades que ofrecía la XO" (M10)

"Después, hacíamos intercambios entre los compañeros. No contábamos lo que íbamos haciendo. Eso me ayudó."(M5)

A modo de expertos navegantes en terrenos desconocidos, se hacen a la aventura que les sugiere conocer nuevas formas de enseñar y en todos los casos observamos que el deseo es el motor principal que mueve a los docentes. Otra vez volvemos al plano ético y moral de la enseñanza.

Estas construcciones transitan a través de concepciones que poseen los docentes sobre los computadores del Plan Ceibal: la XO como cuaderno digital (M1), o fuente de información y actualización (M5), o de puerta a la cultura y al conocimiento (M4).

"Manejar los diarios también...manejar la información... Entonces tenés información, podés llegar a información actualizada también...” (M5)

"Porque es una herramienta que permitiría tener un acceso ilimitado a la información y que puede aumentar la calidad de la enseñanza y la cantidad de saberes que además puede tener una proyección histórica por la costumbre o el buen manejo que tengan una generación" (M4)

Pudimos observar, en algunos de estos docentes, que van modelando sus prácticas de tal manera que crean un estilo personal que las caracteriza. Por ejemplo, la maestra M1 a la que visitamos en dos instancias, en su clase para verla trabajar, utilizó la XO como "cuaderno digital" donde los niños plasmaban sus ideas sobre el tema trabajado: Los factores climáticos y su relación con los biomas. Puede surgir la pregunta: ¿Aportó algo distinto el uso de las TIC en la clase? Según lo observado podemos decir que sí. Pudimos ver la totalidad de los niños involucrados en la tarea pero además, la propuesta y la actividad de la XO seleccionadas por la docente, introducían a la clase la posibilidad de trabajar con imágenes diseñadas por los niños o prediseñadas a través de un programa de dibujo llamado Tux Paint y al mismo tiempo si lo necesitaban, acudir a información a través de internet. En las dos clases que visitamos, esta docente abordó el mismo contenido pero desde distintos aspectos y utilizó el mismo estilo de estrategia que luego retomaremos.

Al comienzo de este apartado decíamos que los docentes vuelcan en sus propuestas, conocimiento disciplinar, didáctico y tecnológico pero surge aquí además, un componente importante y es el pensar ese diseño en función de los destinatarios de las acciones. Es así que estos docentes se plantean el modo en que aprenden sus alumnos, cuáles son las necesidades educativas que existen en la sociedad cuya satisfacción comprenden, de manera implícita, es parte constitutiva de su labor. 


\section{Estrategias en zonas de subducción}

Existen propuestas didácticas que se conciben con sólidos fundamentos didácticos y disciplinares pero que son débiles desde el punto de vista tecnológico, por las limitaciones que presentan. En este grupo tenemos dos ejemplos claros: el primer caso, la propuesta de la maestra M1, que consistía en trabajar sobre el contenido "Biomas de América" a partir de la construcción y descripción de imágenes de paisaje.

La maestra plantea preguntas que buscan provocar la reflexión sobre los elementos constitutivos del paisaje de desierto, extraen conclusiones a partir de información geográfica trabajada con anticipación y luego realizan la representación en sus computadoras a través del programa Tux Paint. Este programa es una aplicación que brinda la posibilidad de realizar dibujos, aporta una serie de herramientas como sellos de animales, vegetales, mapas, entre muchos otros.

Los niños realizaron sus diseños, intercambiando ideas y sugerencias sobre las posibilidades que le daba el programa para concretar su objetivo, describir mediante la imagen un bioma americano.

Desde el punto de vista didáctico, es una actividad sumamente potente en la que se problematiza el conocimiento, se reflexiona sobre los fenómenos geográficos y se potencia el aprendizaje colaborativo. Pero, existe un factor que surge de la entrevista realizada luego de la observación y es la falta de respaldo de esas actividades en un sistema de memoria más duradero.

“... ese es el gran problema que hay. La imposibilidad de registrarlo... Es un obstáculo que los desmotiva a ellos y a los docentes, también... Hemos intentado, fundamentalmente, con el tema de textos, de hacerlo mediante una grabación en pendrive, pero cuando lo pasamos a la computadora convencional que tenemos en nuestros hogares que tanto puede ser con sistema Ubuntu como con el sistema XP, te sale con un código que es con cuadraditos, triangulitos, por lo tanto no lo podemos leer..."

Entonces parte del trabajo realizado por los niños puede perderse por las restricciones que existen hoy para llevar un respaldo de lo realizado. Es así que el empleo de las XO, como "cuaderno digital" no está siendo provechoso, y nos referimos a cuaderno en el entendido de que es un documento pedagógico que guarda cuidadosamente elementos que describen el proceso que va realizando el niño en la construcción del conocimiento.

Por otro lado, la falta de conocimientos en el plano tecnológico y en este caso, las posibilidades de almacenamiento de la información es una limitante más.

El segundo ejemplo alude a la observación realizada; la maestra (M5) aborda el contenido "Las desigualdades entre bloques económicos. Las desigualdades entre los países del mundo. Razones y criterios para su denominación"2.

En cuanto a la estructuración de la clase pudimos observar distintos momentos. Primero, al igual que la docente anterior, hace una presentación de la temática a trabajar a través de preguntas y revisión de conceptos previos. Luego introduce la herramienta tecnológica cuya presencia es clave para la concreción del objetivo que se trazó ${ }^{3}$ ya que la utiliza como fuente de información para analizar los indicadores económicos en grupo; más adelante retoma los análisis realizados por los niños en forma grupal y propone cinco países e indicadores en un cuadro de doble entrada a través del cual pudieran establecer comparaciones. 
La aplicación o actividad, elegida para la clase fue "Navegar" que es básicamente un navegador de internet. La propuesta era ingresar a la página web de la "Guía del mundo"4 donde hay información geográfica de tipo estadístico. Desde el punto de vista didáctico posee una gran riqueza, ya que como plantea Gurevich (2001), introduce estrategias de búsqueda de información significativa para el análisis de la problemática del Desarrollo. Durante esta parte de la actividad, surgieron algunos obstáculos.

A3- Maestra pero está media trancada.

M5- Bueno.

A7- Se tranca.

A1- Maestra...

A3- ¡No! Se cierra, otra vez.

El obstáculo que observamos aquí, fue que en determinado momento, cuando los niños estaban comenzando a conectarse no podían ingresar al sitio por la demanda que representaba sobre el servidor ${ }^{5}$ de internet de la escuela. Los alumnos comentan a la docente: "La antena no da para todos".

Y una alumna que llega de fuera del aula les dice que todas las clases están usando las XO.

Este aspecto tecnológico no se tuvo en cuenta en el diseño de la actividad y configuró un obstáculo en el desarrollo de la misma. ¿Qué hubiera pasado si no lograban acceder a la página web? ¿Existían otras alternativas?

Si bien, luego de algunos minutos todos habían logrado acceder a la página de la Guía del Mundo, pudo haber pasado lo contrario si la demanda al servidor hubiese sido mayor.

Las dos docentes plantearon algunas de estas limitaciones en las entrevistas: falta de respaldo de lo que realizan en la XO (M1, entrevista posterior a la observación); imposibilidad de imprimir los trabajos (M1); problemas de conectividad cuando hay mucha demanda (M5). Entonces, conocían los riesgos pero de todas maneras decidieron asumirlos.

Es así, que de manera metafórica hemos llamado a estas estrategias, "estrategias en zonas de subducción" porque a pesar del aparente sosiego que hay en el terreno -consistencias en el terreno didáctico y disciplinar-, ciertas fuerzas pueden generar "movimientos sísmicos" aspectos tecnológicos- de gran magnitud que destruyan en un momento lo que se ha construido.

En este riesgo que asumen las docentes al incluir el recurso tecnológico sin tener en cuenta las limitaciones y los obstáculos que se pueden enfrentar, surgen nuevas interrogantes: ¿Se desdibuja la función de la tecnología en la enseñanza? ¿Hasta qué punto el docente puede permanecer ignorante de los aspectos tecnológicos?

\section{5.a. Estrategias en emergencia - estrategias emergentes.}

Bajo este título, a través de la observación de clases (observación 4 y 6), hemos agrupado estrategias de enseñanza de la Geografía en las que participan las tecnologías pero que han sido concebidas desde débiles sustentos, alejados de las posibilidades reales que ofrece la $\mathrm{XO}$. Todo esto confabula en contra de los planes que se traza el docente generando situaciones que impiden llevarlos a cabo, surgiendo alternativas que vienen a salvar la actividad.

En una de las clases, la docente solicitó al grupo, como tarea domiciliaria del día anterior que instalaran el Google Earth en sus computadores para realizar la actividad que íbamos a observar. Al comenzar la clase surgió que ningún niño del grupo había logrado instalarla.

M4- No pudiste ¿Por qué?

A1- No sé. Porque soy muy desafortunado. 
M4- Intentaste varias veces y Google Maps ¿y tampoco?

A1- Tampoco.

(...)

A4- Porque en realidad, nosotros, cuando vamos a buscar, te aparece para Windows XP.

M4- ¿Te aparece para qué?

A4- Para Windows XP.

M4- ¿Y la qué dice para XO tampoco?

A4- No hay para XO.

(Fragmento de Observación 4)

El desconocimiento de la docente sobre la imposibilidad de usar el Google Earth en las XO es evidente en este fragmento. Es así que la docente tuvo que cambiar la actividad que había planificado, en el momento. Con mucha rapidez surgieron ideas que posibilitaron a la docente tratar el contenido ("Influencia de la tecnología en la evolución de la cartografía"6) por otras vías que no pensaba.

Utiliza el recurso de la navegación en internet para la búsqueda de planos e imágenes satelitales de Montevideo y de Uruguay con los que a través de la observación lleva a los niños a la reflexión sobre la importancia de las tecnologías en el desarrollo de cartografía, realizando un análisis histórico de los cambios.

Unos días después, en la siguiente clase observada, la maestra sugiere comenzar la clase mirando un video publicado en YouTube. Aquí vuelve a surgir un problema, y es que el servidor de Ceibal restringe la entrada a la dirección de este sitio web. Por lo tanto, nuevamente tuvo que crear estrategias "emergentes" para sobrellevar la situación.

En las dos oportunidades, la maestra elaboró estrategias que desde el punto de vista tecnológico, eran imposibles de llevar a cabo en las XO. Seguramente, en otro tipo de computadora, estas mismas propuestas didácticas hubieran sido altamente significativas para los niños pero no se tuvo en cuenta, quizá por desconocimiento o falta de capacitación en el uso de las $\mathrm{XO}$, uno de los aspectos principales de la planificación de este tipo de propuestas didácticas, que es el tecnológico. La docente nos decía en la entrevista "Y nada...Y enseguida hubo el problema de que no estábamos formados nosotros para poder utilizar la herramienta como se debería porque no teníamos ningún curso a la vez que la implementación. Como un desfasaje." (M4)

En el primer ejemplo, era imposible instalar el Google Earth por la capacidad de memoria que poseen las $\mathrm{XO}$, y en el segundo ejemplo, Ceibal tiene una protección contra el ingreso a algunos sitios, no permitiendo acceder a ellos; un caso es YouTube. Por lo tanto es imprescindible tener claro elementos de esta índole para que se puedan llevar a cabo estrategias de enseñanza mediadas por tecnología.

\section{5.b. Estrategias volcánicas.}

Hemos llamado a estas estrategias "volcánicas" porque surgen desde el convencimiento interno de que las tecnologías pueden favorecer la creación de situaciones de aprendizaje en las que exista una participación activa de los alumnos aportando nuevos conocimientos (Meirieu, 1992, 147); y "emergen a la superficie", el escenario del aula, generando impactantes resultados. 
En esta categoría hemos ubicado la propuesta de enseñanza de una maestra de Florida (M8), y explicitamos el lugar porque consideramos que el tiempo que llevan trabajando, ensayando propuestas y actividades, ha sido un elemento importante en la elección de las estrategias de enseñanza con implementación de las tecnologías.

La docente retoma conceptos trabajados anteriormente referentes a la población a través de preguntas que guían a los niños para ir construyendo un análisis.

"Vamos a retomar un poquito la actividad que estábamos trabajando. Nosotros veníamos trabajando con lo que era población, ¿recuerdan?...”

"Ahí está, ¿qué era la tasa de natalidad?"

"¿Cuándo decíamos que era una población vieja?..."

"¿Qué hablamos de la esperanza de vida?..."

"La población, ¿va a aumentar o disminuir?"

“¿La población está relacionada con la superficie en todos los casos?”(M8)

Estas y otras preguntas estructuraron el diálogo que se generó en la clase para luego, a partir de gráficas que tenían en el libro y otros datos que les fue dando la maestra, analizaron la dinámica del crecimiento de la población.

Analizaron datos estadísticos que tenían en el libro sobre este tema, y posteriormente solicitó a los alumnos que realizaran una gráfica de población con las expectativas al 2050, usando un programa del tipo Hoja de Cálculo llamado Social Calc, y que la compararan con la gráfica de población actual que tenían ya hecha en la XO. Explicó el procedimiento para el cálculo de porcentajes y luego permite a los alumnos realizar la actividad en grupos de a dos. Para finalizar comparten las gráficas con los compañeros.

El intercambio que se dio entre los niños, y entre estos y la docente fue perfilando el análisis del crecimiento de la población. La utilización de las tecnologías en esta actividad permitió facilitar la interpretación de datos a partir de gráficos que, haberlos hecho de otro modo hubiera requerido mucho tiempo y se perdería el sentido de la actividad que era el análisis y no la construcción de gráficos. La maestra nos decía en la entrevista:

"iLas gráficas, que antes estaban horas!" (M8)

Además nos comentaba que cuando recibieron las computadoras, en el año 2007, no tuvieron cursos de capacitación por lo que desarrollaron redes entre los docentes que les permitieron aprender con los compañeros, y autoformarse en el uso de las XO.

"Primero, mucho miedo, porque yo no sabía ni como se abrían esas maquinitas... después fuimos aprendiendo." (M8)

Podemos afirmar que en esta maestra, el deseo de conocer y de incluir de forma adecuada el uso de las XO en las estrategias de enseñanza fue el motor que impulsó los sucesivos ensayos que la llevaron a hacer una "inclusión genuina" (Maggio, 2005) de las tecnologías.

Por lo que indican los datos, consideramos que el tiempo ha sido otro factor determinante. Probablemente hace tres años atrás, esta maestra no habría hecho esta actividad con las XO para enseñar Geografía, quizá porque los docentes también necesiten de tiempo para pasar por diversas etapas en su aprendizaje.

En las entrevistas que realizamos a otras maestras, M1, MPG, M10, surge la variable tiempo de la siguiente manera:

"En Geografía específicamente, yo si en este momento tuviera que volver a hacer el libro le agregaría más trabajo en XO en vista de lo que he estado trabajando con los chiquilines el año pasado y este." (M1)

"Introduciría algunas aplicaciones que antes no teníamos", afirmó M10 respecto a cambios que haría en el libro de texto que elaboró con otras colegas. 
"Ya te digo, este año más, con respecto al año pasado y supongo que después más todavía. Te da una cantidad de posibilidades de investigar..." dice MPG sobre el cambio en sus prácticas de enseñanza.

Es por esto que afirmamos que el tiempo que transitan los docentes empleando las $\mathrm{XO}$, es una variable importante en la calidad de las estrategias que planifican.

\section{5.c. Estrategias carentes de Norte.}

En este grupo ubicamos a las estrategias cuyos objetivos se alejan de la enseñanza de la Geografía, y las decisiones en torno al empleo de las TIC no fueron pertinentes, no aportando a la construcción de los conocimientos geográficos. Parecen no tener un rumbo claro.

De estas estrategias encontramos dos ejemplos: la primera, propuesta por la maestra (M9), consistía en contestar un cuestionario referido a varios conceptos Geográficos y Geológicos, con apoyo del navegador de internet de la XO para acceder a la información. Esta actividad no se pudo realizar de la forma propuesta porque el servidor de Ceibal de la escuela no estaba funcionando, por lo que la maestra solicitó a los niños que usaran los libros de Ciencias Sociales para responder las preguntas que había traído impresas.

La segunda propuesta consistió en trazar un recorrido sobre un plano de Colonia del Sacramento. El plano debían obtenerlo de Google Maps y tomar una captura de pantalla que luego editarían en el programa Dibujar de la XO. Aparentemente, si vemos la propuesta alejada del acontecer del aula podría decirse que era muy válida desde el punto de vista de la Geografía. Los niños debía aplicar determinados conocimientos y estrategias que les permitieran trazar una ruta, pero a lo largo de la actividad surgieron varios elementos que nos hicieron ver cuán alejadas estaban las estrategias de enseñanza, del propósito de la actividad planteada por la docente.

En este caso, al conectarse tantos niños y al requerir el Google Maps cierta velocidad de bajada de datos hizo imposible obtener allí la imagen que necesitaban. Un niño propuso un sitio de internet donde podían encontrar un plano que era posible ver. Para lograr esto, pasaron cuarenta minutos en un ambiente caótico donde muchos niños, desinteresados de la actividad estaban distraídos en conversaciones y juegos. Unos pocos niños persistieron, a pesar del clima del aula y los obstáculos en el acceso a la imagen del plano, y llegaron a representar el recorrido.

Como decíamos al comenzar el análisis de esta clase, surge la duda de cuál era el contenido real que se estaba enseñando. Gran parte de la actividad estuvo dedicada al manejo de la XO y no a la localización geográfica. En segundo lugar, el uso de la XO se transformó en un obstáculo para la tarea planteada. Seguramente, usando otro tipo de plano habrían podido realizar la ruta que se les indicó de una forma más sencilla y poniendo atención a los elementos del lenguaje cartográfico y la localización, además de distribuir de modo adecuado el uso del tiempo en los distintos momentos de la actividad.

En cuanto al primer ejemplo, de la maestra M9, consideramos que la elección del recurso tecnológico para llevar a cabo la actividad, fue errónea más allá de que no se pudo llevar a cabo por problemas de conectividad: el cuestionario que presentó la maestra apelaba a aprendizajes de tipo memorístico y posiblemente, los niños que gozaran de buena memoria o de suspicacia para mirar la respuesta del compañero, lo resolverían sin mayor dificultad. Y en el caso del último ejemplo, el empleo de las TIC se convirtió en un obstáculo para la concreción de la actividad propuesta. 
En estos dos casos, probablemente las intenciones de las docentes fueran buenas, porque partimos del supuesto que todos pretenden enseñar algo en las acciones que llevan a cabo dentro de sus aulas. Pero evidentemente, la elección y tratamiento de los contenidos por la primera, y la elección del recurso por la segunda, no fueron cuidadosamente pensados para la concreción de sus metas.

\section{Conclusiones}

Esta investigación fue el resultado de una mirada sobre las prácticas de enseñanza que, con las tecnologías y a través de ellas, buscan alcanzar objetivos que persiguen el desarrollo de conocimiento geográfico. Describe y analiza dichas prácticas en un contexto que ha sido conmovido por la irrupción del Plan Ceibal el cual incorporó a las aulas una computadora por niño y por docente. Realizada a un año de la finalización de la etapa de la entrega de XO en Educación Primaria, deja abierta muchas interrogantes.

De acuerdo a De la Torre (2000), la correcta elección de las estrategias guiará al docente por el camino del cambio en los individuos, en las instituciones y en la sociedad. Lejos estamos de indicar caminos correctos y opinar insidiosamente sobre los caminos que eligieron nuestros protagonistas, quienes participaron con generosidad de la muestra. Por ello aportaremos unas reflexiones en torno a la tarea de enseñar Geografía hoy, con las XO.

Estamos convencidos que hay una variedad muy amplia de estrategias y éstas están estrechamente ligadas a la formación profesional, actualización, capacitación tecnológica y al deseo, que traen al aula los docentes. Todos estos factores inciden directamente sobre las posibilidades que ofrece la herramienta tecnológica en la enseñanza de la Geografía.

La mayoría de los docentes entrevistados han seguido procesos de autoformación y formación continua que les han permitido un desarrollo profesional teóricamente óptimo y en consonancia con las necesidades educativas de sus alumnos. Por lo tanto, sería erróneo afirmar que estos maestros no cuentan con las competencias necesarias para diseñar situaciones de enseñanza eficaces.

Sin embargo, las TIC se introducen como una suerte de variable no prevista: un nuevo elemento a incorporar en la planificación de las actividades áulicas. Parece ser que la competencia didáctica de los maestros no contempla el uso de las nuevas tecnologías. Esto podría relacionarse con propuestas de formación en servicio que sólo recientemente han considerado el uso didáctico de las TIC como contenido. Es aquí donde surge una nueva interrogante que podrá ser develada en otras investigaciones: ¿Cómo hacer para que los docentes que se mantienen al margen de las tecnologías, ignorando la velocidad de los cambios y su importancia en el acontecer de las aulas, puedan ser protegidos de ese "aislamiento"? ¿Cómo hacer para acortar brechas entre docentes y tecnologías?

Por otra parte, pudimos observar que las formas de usar las tecnologías esbozan estilos que se presentan desde distintos enfoques: algunas desde el entendido de la XO como cuaderno digital, otras, emplean la XO como puerta de acceso a la información, y otras como medio para analizar esta información. Desde estos enfoques, ellas pueden venir a ser fuente de información a través de la que se desarrollen estrategias que permitan la comprensión de los fenómenos geográficos; pueden ser el medio por el que los niños impriman las huellas de de sus recorridos de aprendizaje. Pueden ser potentes herramientas en la adquisición de destrezas geográficas como la lectura de cartografía, localización georeferenciada, el análisis de datos estadístico entre otros, pero no por sí solas adquieren esta condición; el docente es quien le atribuye significado al diseñar las estrategias que utilizará para lograr sus objetivos.

A partir de las observaciones y las entrevistas que realizamos, pudimos concluir que la 
Geografía es un campo que debe fortalecerse desde la especificidad de la disciplina y desde los aportes didácticos actuales que utilizan las tecnologías en la enseñanza como forma de mejorarlas y promoverlas.

Por lo tanto, lo disciplinar y lo tecnológico son elementos a considerar en la formación docente para promover una reflexión profunda sobre las estrategias de enseñanza de la Geografía que devenga en buenas prácticas.

De esta investigación surge también la necesidad de revisar con atención la pertinencia del recurso tecnológico valorando los aportes reales que hace a la construcción del conocimiento. Del trabajo de campo surgió la interrogante sobre el valor que aporta el uso de la XO en las clases de Geografía. Pudimos constatar que en algunos casos no solamente no aportó, sino que ofició de obstáculo, a veces de tal magnitud, que desbarató una estrategia planificada; y en otros casos vino a ser la principal herramienta para la enseñanza del contenido seleccionado.

En cuanto a las estrategias pudimos categorizar los siguientes tipos:

"Estrategias en terrenos de subducción", son las que se conciben desde el punto de vista didáctico, con profundos fundamentos, pero por las inconsistencias en la formación sobre las tecnologías crean incertidumbres que generan riesgos. Alumnos que pierden el interés por la utilización de la XO porque sus tareas se borran y no tienen respaldo, o porque no es posible imprimirlas. Docentes que al enfrentarse a tantos obstáculos que surgen por la falta de conocimiento de la herramienta, se desmotivan, dejan fuera de sus aulas a las TIC. Pero en este grupo predominan las decisiones de tipo metodológico que implican riesgos, incertidumbres. Los docentes ya saben que se van enfrentar a determinados problemas, pero de todas maneras se arriesgan, aprovechando al máximo las potencialidades que tiene la XO para las actividades de enseñanza.

"Estrategias en emergencia - estrategias emergentes", son las estrategias de enseñanza de la Geografía en las que participan las tecnologías, pero que han sido concebidas desde débiles sustentos, alejados de las posibilidades reales que ofrece la $\mathrm{XO}$, conspirando en contra de los planes que se traza el docente, generando situaciones que impiden llevarlos a cabo y dando como resultado el surgimiento de otras estrategias alternativas que vienen a salvar la actividad.

"Estrategias volcánicas". Son aquellas que se producen desde convicciones profundas sobre las potencialidades de la tecnología en la enseñanza y desde fuertes sustentos didácticos y tecnológicos. Ellas producen cambios cognitivos en entornos favorecedores. Las tecnologías aquí vienen a ser un puente entre el niño y el conocimiento.

"Estrategias carentes de Norte". Estas estrategias son aquellas que han sido elaboradas sin respetar la coherencia interna de la disciplina, y el uso del recurso tecnológico no ha sido pertinente ni ha aportando ningún beneficio en la construcción de los conceptos geográficos. "La enseñanza es estéril si no elabora situaciones de aprendizaje en donde el alumno pueda realizar una elaboración activa, es decir, una integración de nuevos datos en su estructura cognitiva" (Meirieu, 1992, 147).

Los hallazgos de esta investigación además de responder a los objetivos que nos planteamos al principio, despiertan nuevas preguntas, presentan nuevos problemas. Podrán ser tratados o no en futuras investigaciones. Por nuestra parte quedan planteadas a lo largo de este trabajo. 


\section{Bibliografía}

Buckingham, D. 2008. "Más allá de la tecnología. Aprendizaje infantil en la era de la cultura digital”. Manantial. Buenos Aires.

Buzai, G. 2004. "Geografía Global. El paradigma Geotecnológico y el espacio interdisciplinario de la interpretación del mundo del siglo XXI”. Ed. Lugar. Bs. As.

Eggen, p. Kauchak, d. 2000. "Estrategias docentes. Enseñanza de contenidos curriculares y desarrollo de habilidades de pensamiento." Fondo de Cultura Económica. Bs. As. $1^{\text {a }}$. Reimpresión.

Fullan, M. 2002 “Los nuevos desafíos del cambio educativo”. Octaedro. Barcelona.

Gimeno Sacristán, J. PÉREZ GÓMEZ, A. 1995 “Comprender y transformar la enseñanza”. Ed. Morata. Madrid.

Levis. Gutiérrez Ferrer. 2000 “¿Hacia la herramienta educativa universal? Enseñar y aprender en tiempos de internet" Ciccus. Bs. As.

Litwin, E. 2008 a. “Las configuraciones didácticas”. 4ta. Reimpresión. Paidós. Bs. As.

Meirieu, P. 1992. “Aprender sí, pero ¿cómo?”. Octaedro. Barcelona.

Pardinas, F. 1996. "Metodología y técnicas de investigación en ciencias sociales". Siglo XXI. Méjico.

Taylor, S. J. Bogdan, R. 1987. "Introducción a los métodos cualitativos de investigación”. Paidós. Bs. As.

Wittrock, M. 1989. "La investigación de la enseñanza. II. Métodos cualitativos de observación". Paidós. Barcelona.

Woods, P. 1998. “Investigar el arte de la enseñanza: el uso de la etnografía en educación”. Paidós. Barcelona.

Zappettini. Zilio. Et al. 2008. "Los sistemas de información Geográfica - SIG - en la enseñanza de la Geografía". Disponible en Internet: < http://es.scribd.com/doc/.../3289Zappettini-Maria-Cecilia1>

Otras fuentes:

MUSEO DE ARTE THYSSEN - BORNEMISZA. www.Museothyssen.org 


\section{Notas}

1. Para el análisis de las estrategias docentes empleamos, lo que llamamos "metáfora geográfica". Utilizamos pinturas de grandes artistas que reflejan aspectos que se identifican en los docentes de la muestra.

2. Contenido programático que aparece en la planificación de la docente.

3. El objetivo que la docente plantea en la planificación es: "Reconocer y comparar los indicadores sociales, económicos, demográficos que se utilizan para denominar los bloques económicos"

4. La maestra escribe en el pizarrón la dirección del sitio: "http://www.guiadelmundo.org.uy/cd"

5. Servidor es un ordenador remoto que provee la información solicitada por los navegadores de otros equipos.

6. Al finalizar la clase le consultamos sobre el contenido abordado y nos escribió éste en la ficha de observación. Ver en anexo.

* Fecha de recibido: 1\%02/2012

Fecha de aceptado: 9/04/2012

** Master en Educación y Diploma en Educación, Universidad ORT Uruguay. Maestra en Educación Común, IINN. Docente adscriptora, CEIP. Maestra Dinamizadora del Plan Ceibal, Inspección Nacional de Escuelas de Práctica. Maestra de grado, C.L.E.B. 